remission. There was no association between reasons for discontinuation of the first therapy because of primary or secondary failure and adverse effects $(3,4 \pm 0,9$ vs $3,75 \pm 1,11$ vs $3,86 \pm 1,3, p=0,6$ ) with the number of treatments received.

Conclusions: In our biologic therapy RA-PAZ cohort, we found a subgroup of younger pts, with a more systemic phenotype of the disease and a higher disease activity,who required a prompter biological therapy initiation. This subgroup of pts is more susceptible to biological treatment failures. The development of ADA after the first biological agent was also associated with the need to use more biologics. Disclosure of Interest: None declared

DOI: 10.1136/annrheumdis-2017-eular.5494

\section{AB0199 METHOTREXATE RESPONSE IN EARLY RHEUMATOID ARTHRITIS ASSESSED USING A SOMAMER PROTEOMIC ASSAY}

C. Hitchon, V. Spicer, X. Meng, A. Gao, H.S. El-Gabalawy, J. Wilkins. University of Manitoba, Winnipeg, Canada

Background: Optimizing treatment in early rheumatoid arthritis (ERA) improves clinical outcomes. Developing approaches that would allow for accurate outcome predictions would be useful. We examined the possibility of employing SOMAscan to identify biomarkers that predict treatment response.

Objectives: To define methotrexate (MTX) 6 month treatment associated response protein changes using SOMAscan.

Methods: Sera from 14 Disease Modifying Antirheumatic Drug (DMARD) naive ERA patients at baseline (PRE) and after six months of MTX (POST) were analyzed using SOMAscan, an aptamer based assay that offers simultaneous relative quantitation of 1310 proteins. RA activity was measured by DAS28ESR3var abbrev DAS3; RF and ACPA were measured at baseline. SOMAmer intensity data was log2 transformed and differences ( $D=P O S T$-PRE) clustered using undirected hierarchical self-organization. Kolmogorov-Smirnov differential analysis determined SOMAmers contributing to these populations at $p<0.05$. Potential processes associated with these SOMAmer regulation groups were identified using an in-house biological enrichment tool.

The potential for SOMAmers to predict treatment response was also explored; for this we defined a fractional clinical response metric dDAS3 $=$ (DAS3_POSTDAS3_PRE)/DAS3_PRE. We then selected a population of proteins ( $n=3$ to avoid over-fitting) with PRE expression levels best correlating to dDAS3. These three PRE expression values formed a weighted average, with weighting coefficients optimized by a simple Monte-Carlo method. We included this weighted average with clinical variables in logistic regression models, where 6 month DAS3 was the dependent variable.

Results: Clustering gave two populations of 6 and 8 patients (POP0, POP1) with mean delta DAS3 values of -1.71 and -0.46 respectively. In POPO compared to POP1, 113 proteins were upregulated and 121 proteins were downregulated. The upregulated proteins were involved in VEGF signalling and platelet activation. The downregulated proteins were involved in regulation of immune response, cellular response to TNF and cytokine -cytokine receptor interactions. The fractional change dDAS3 correlated well with the treatment response panel $\left(R^{2}=0.8645\right.$; $\mathrm{p}=6.8 \mathrm{e}-5)$, with the caution that expression values of the 3 best-correlating proteins exhibited low coefficients of variation $(<0.1)$. However, these proteins did reflect RA responses or inflammation. This weighted sum was also independently associated with treatment response in regression models including baseline DAS3 (or components) and RF/ACPA.

Conclusions: This pilot study suggests that high content proteomic approaches such as SOMAscan may be useful for developing prediction tools of patient responses to treatment. Extension of this work into a larger patient population is ongoing.

References:

[1] Journal of Statistical Software, 8(18) 1-4, 2004.

[2] Nature Protocols, 4(1) 44-57, 2009

Disclosure of Interest: None declared

DOI: 10.1136/annrheumdis-2017-eular.1747

\section{AB0200 MUSCULOSKELETAL ULTRASOUND ADDED TO ROUTINE EVALUATIONS OF RHEUMATOID ARTHRITIS PATIENTS HAS A DIFFERENT IMPACT ON THE TREATMENT PROPOSAL DEPENDING ON PHYSICIAN EXPERIENCE}

C. Sifuentes ${ }^{1}$, I. Contreras-Yáñez ${ }^{1}$, M. Gutíerrez ${ }^{2}$, V. Pascual ${ }^{1} .{ }^{1} /$ mmunology and Rheumatology, Instituto Nacional de Ciencias Médicas y Nutrición Salvador Zubiran; ${ }^{2}$ Instituto Nacional de Rehabilitación y Ortopedia, México, D.F, Mexico

Background: Disease activity (DA) is the most important factor in the treatment decision/monitoring during rheumatoid arthritis (RA) patient's follow-up. In routine clinical practice, it is recommended to regularly evaluate DA level from patients with RA. Musculoskeletal ultrasound has been suggested to add value to establish the level of DA; evaluations that assess a reduced number of joints, as the German ultrasound score of 7 joints (GUS-7) are easy to incorporate in clinical practice (1).

Objectives: To explore the real impact of GUS-7 in the treatment recommendation to RA outpatients, currently attending an Early Arthritis Clinic (EAC). The primary objective was to determine the proportion of patients in whom treatment recommendation differed after GUS-7 examination. We additionally tested the variations of GUS-7 impact according to the physician's experience (senior rheumatologist [SR] vs. trainee in rheumatology [TR]).

Methods: A sample size of 84 evaluations was calculated to achieve the primary objective. Eighty-seven consecutive and randomly selected RA outpatients were invited to participate; 2 patients denied because of administrative reasons and the 85 patients left underwent 170 assessments ( 85 each by the SR and the TR). At first, both physicians (blinded to each other evaluations) performed a clinical evaluation that included DAS28 scoring and recommended a RA-treatment. Then, patients underwent GUS-7 by a blinded (to clinical evaluations) rheumatologist that additionally determined the sonographic disease activity. In the final step, the TR and the SR integrated the US findings to their previous evaluation and reviewed their prescription; GUS-7 findings, pre- and post-GUS-7 treatments were recorded on standardized formats. Patients received final recommendation only from the SR. All the patients signed informed consent and were instructed about the process. Descriptive statistics was used.

Results: Patients were primarily middle-aged [(mean \pm SD) $45.13 \pm 12.4$ years] female $(91.4 \%)$, with $($ mean \pm SD) disease duration of $7.5 \pm 3.9$ years. Most of the patients $(69.2 \%$ according to TR and $71.8 \%$ to SR) were in DAS28-ESRremission, although the four levels of DA were represented. Agreement between both physicians was good (Kappa: $0.82, p \leq 0.001$ ). Most frequent GUS-7 findings were grey scale synovitis in at least one joint in $98.8 \%$ of the patients, among whom $22.6 \%$ had Power Doppler activity (PD); one third of the patients had tenosynovitis although few (12\%) had PD; erosions were detected in $38.8 \%$ of the patients.

In 34 of 170 clinical scenarios (20\%), GUS-7 findings modified treatment; treatment changes (after GUS-7 findings were incorporated to clinical findings) consisted of an increase in $24(70.6 \%)$ scenarios, a decrease in $8(23.5 \%)$ and joint injection with corticosteroids in $2(5.9 \%)$. Interestingly, 24 of the 34 clinical scenarios with GUS-7 treatment impact were performed by the TR vs. 10 performed by the SR: $70.5 \%$ vs. $29.5 \%, p=0.01$. Treatment changes (increase, decrease and joint injection) were similar among both specialists.

Conclusions: In routine clinical practice of RA patients, GUS-7 assessments impacted treatment decision in $20 \%$ of the patients; the impact was stronger among TR than among SR.

References:

[1] Backhaus et al. Arthritis Rheum 2009; 61: 1194-201.

Disclosure of Interest: None declared

DOI: 10.1136/annrheumdis-2017-eular.1435

\section{AB0201 INFLUENCE OF SIGA ON CLINICAL ACTIVITY MARKERS IN SPA PATIENTS WITH NON-RADIOGRAPHIC AND PERIPHERAL COMPROMISE}

C. Romero-Sanchez ${ }^{1}$, F. Salas-Cuesta ${ }^{2}$, I. Arias $^{3}$, J.M. Bello-Gualtero ${ }^{4}$ W. Bautista-Molano ${ }^{1}$, D. Herrera ${ }^{3}$, D. Castillo ${ }^{5}$, R. Valle-Oñate ${ }^{1}{ }^{1}{ }^{1}$ Department of Rheumatology and Inmunology, Hospital Militar Central; ${ }^{2}$ School of Medicine, Universidad Militar Nueva Granada: ${ }^{3}$ Universidad Javeriana: ${ }^{4}$ Department of Rheumatology and Inmunology, Hospital Militar Central, Bogotá, Colombia; ${ }^{5}$ UIBO Institute, Universidad El Bosque, Bogotá D.C., Colombia

Background: There are previous evidence about inflammatory signs related with the intestinal mucosa in spondyloarthritis patients with seronegative arthritis and them relation with articular inflammatory activity. It is uncertain the role of these serological markers on the inflammatory/clinical activity in patients with SpA

Objectives: To establish the relationship among activity variables and indices, and soluble markers associated to mucosal associated lymphoid tissue in a group of SpA patients.

Methods: Patients were selected by rheumatologists with the ESSG criteria. Levels of SIgA, IgA, IgA Chlamydia trachomatis, Shigella spp, Yersinia ssp, Campilobacter ssp and Salmonella ssp, CRP,ESR,HLA-B27,BASDAI,ASDASCRP and ASDAS-ESR were determined. A principal components analysis (PCA), Poisson Regression and multiple correspondence analysis were performed to find relationships between clinical and laboratory variables and SIgA. This study was approved for Ethics Committee.

Results: 46 patients were included $(78.2 \%$ males with a mean age $34.8 \pm 12.3$ years). It was reported at least one gastrointestinal sing in $69.2 \%$ of patients:abdominal bloating $(45 \%)$, abdominal pain $(43 \%)$; all patients showed at least one musculoskeletal symptom, $69.5 \%$ enthesitis, $63 \%$ inflammatory back pain and $58.6 \%$ arthritis, as well as $43.4 \%$ previous infection and $47.8 \%$ presented HLA-B27.The PCA showed three principal factors which cover a contribution of $82.2 \%$ to explain the SIgA variation. The ASDAS-CRP, ASDAS-ESR, BASDAI variables which provide the $47.12 \%$; the regression model shows an inverse association among SIgA and BASDAI (prevalence ratio (PR):0.43, 95\% Cl:0.26-0.70 $\mathrm{p}=0.001$ ), ASDAS-CRP (PR:0.72, 95\% Cl:0.24-0.95 $\mathrm{p}=0.021$ ) and ASDAS-ESR (PR:0.69, 95\% Cl:0.39-0.95 p=0.007); however, a risk was demonstrated among BASDAl and Yersinia IgA (PR:1.68 95\% Cl:1.03-2.74 p=0.036) and between ASDAS-CRP with HLA-B27 (PR:1.62 95\% Cl:1.18-2.19 p=0.0002). There was a relationship between the absence of clinical activity (ASDAS-CRP, ASDAS-ESR and BASDAI), previous infection, Yersinia IgA with SIgA Q1 $(27.8-43.0 \mathrm{ug} / \mathrm{mL})$; the presence of arthritis, Salmonella IgA, and high levels of CRP and ESR were related with SIgA Q2; SlgA levels among (Q3)12.2-18.0 ug/mL were associated with inflammatory back pain, obesity and Salmonella $\lg A<1 / 1600$. High scores 